\title{
Surgical and Non-Surgical Management and Treatment of Glioblastoma: I. Primary Tumors
}

\author{
Alain L Fymat* \\ International Institute of Medicine \& Science, USA
}

Submission: October 22, 2017; Published: December 08, 2017

*Corresponding author: Alain L Fymat, International Institute of Medicine and Science, California, USA, Tel: (760) 485-9149;

Email: alain.fymat@fiimas.org

\begin{abstract}
Glioblastoma, the most common primary brain tumor in adults, remains an unmet need in oncology. Its management and treatment begin with an understanding of this tumor's morphology, biological types, risk factors, and prognosis. The various surgical and non-surgical modalities devised so far for primary tumors and their metastases are presented and discussed either singly or in combination. For each such therapy, the treatment results obtained in clinical trials and other reported practices are also summarized and discussed. Historically, chemotherapy has provided little durable benefit with tumors recurring within several months, even in the case of more accessible tumors located outside the brain, let alone within the brain behind its protective barriers. More effective therapies involving other options are required either in isolation or more likely in combination. Of these other options, the following are considered here at some length: surgery, conformal radiotherapy, boron neutron therapy, intensity modulated proton beam therapy, antiangiogenic therapy, alternating electric field therapy, immunotherapy without neglecting palliative therapies. A companion paper will discuss the important case of recurring tumors.
\end{abstract}

Keywords: Antiangiogenesis Therapy; Astrocytoma; Bevacizumab; Blood Brain Barrier; Boron Neutron Capture Therapy; Cediranib; Cilengitide; Conformal Radiotherapy; Dexomethasone; Electric Field Therapy; Epidermal Growth Factor Receptor; Glioblastoma; Glioblastoma Multiform; Gliomatosis; IDH1 Mutation; Li Fraumeni Syndrome; Molecular Tumor Analysis; MGMT Methylation; Neurofibromatosis; Proton Beam Therapy; Radio chemotherapy; Recursive Partitioning Analysis; Stem-Like Cancer Cells; Stevens-Johnson Syndrome; Surgery; Temozolomide; Tuberous Sclerosis; Turcot Syndrome; Von Hippel-Lindau Syndrome

Abbreviations: AIFEFT: Alternating Intermediate Frequency Electric Field Therapy; BBB: Blood Brain Barrier; BNCT: Boron Neutron Capture Therapy; BPB: Brain Protective Barriers; CNS: Central Nervous System; CRT: Conformal Radio Therapy; CSF: Cerebro Spinal Fluid; DFS: DiseaseFree Survival; DNA: Deoxyribo Nucleic Acid; EGFR: Epidermal Growth Factor Receptor gene; EGFRV: EGFR Variant; EVGF: Endothelial Vascular Growth Factor (a pro-angiogenic cytokine); FDA: (U.S.) Food \& Drug Administration; GB: Glio Blastoma; GBM: Glioblastoma Multiform; GBSCL: GB Stem-Cells Like ; IMPBT: Intensity Modulated Proton Beam Therapy; KPS: Karnofsky Performance Score; MGMT: 06-alkylguanine DNA alkyltransferase; MRI: Magnetic Resonance Imaging; MRS: Magnetic Resonance Spectroscopy; MTA: Molecular Tumor Analysis; ODEC: Oxygen Diffusion-Enhancing Compound; OS: Overall Survival; PBC: Population-Based Cure; PFS: Progression-Free Survival (time without tumor progression); RANO: Response Assessment in Neuro-Oncology; ROD: Risk of Death; RPA: Recursive Partitioning Analysis; SCLC: Stem-Cell Like Cancer; TMZ: Temozolomide (brand names: Temodar, Temodal and Temcad); TSC: Tumor Stem Cell

Disorders cited: Astrocytoma (including an a plastic astrocytoma); Brain Tumor; Cancer; Cysts (multiform); Cerebral Edema; Drowsiness; Erythema Multiform; Headache; Hemorrhage; Glioblastoma; Glioblastoma Multiform; Intracranial Pressure; Li Fraumeni Syndrome; Meningeal Gliomatosis; Meningioma; Necrosis; Neurofibromatosis (types 1 and 2); Pleocytosis; Radionecrosis; Stevens-Johnson syndrome; Swelling; Tuberous Sclerosis; Turcot syndrome; Von Hippel-Lindau Syndrome.

Drugs mentioned: 5-Aminolevulinic Acid (a surgery dye); Anticonvulsants; Bevacizumab (a monoclonal antibody with activity against EVGF); Cediranib (an angiogenic inhibitor); Cilengitide (an inhibitor of integrin $\alpha \mathrm{V} \beta 3$ and $\alpha \mathrm{V} \beta 5$ ); Corticosteroids (including anti-convulsant ones); Cytarabine; Dexamethasone (a corticosteroid); Integrin Inhibitors; Phenytoin; Steroids; Temozolomide.

\section{Introduction}

Glioblastoma (GB), also known as Glioblastoma multiform (GBM), is the most common primary brain tumor in adults. It remains an unmet need in oncology. It is the most aggressive and most common brain cancer, the second most common after meningioma, representing $15 \%$ of brain tumors. Its annual incidence is $\sim 3.19$ cases per 100,000 population with the average age at diagnosis being 64 years. It occurs more commonly 
in males than females. The survival rate is $\sim 1$ year, and only 5 $\%$ of the people affected survive for 5 years [1-4]. Although firstline treatment has been clearly defined since 2005, no standard second-line treatment has yet been determined. Also, while several possible risk factors have been discussed, no prevention strategy is known,

Most treatments cannot eradicate all tumor cells. Thus, surgery is often insufficient given the diffuse nature of the disease; chemotherapy has major limitations because most drugs cannot cross the blood brain barrier (BBB), and penetration into brain cells is limited. In addition, the cells in brain tumors are greatly heterogeneous, which limits the treatment efficacy and explains the high rate of progression of the disease.

The standard treatment consists of (a) surgery (maximal resection) followed by (b) radio chemotherapy (6 weeks of radiotherapy at a dose of 60 Grey [Gy]) together with concomitant chemotherapy with Temozolomide (TMZ) at a rate of $75 \mathrm{mg} / \mathrm{m} 2$ daily) (TMZ is an alkalizing agent used as a first-line treatment for $\mathrm{GB}$ and as a second-line treatment for astrocytomas) and, once radio chemotherapy is complete, (c) adjuvant treatment (a minimum of 6 months with TMZ is started at a dose of 150-200 $\mathrm{mg} / \mathrm{m} 2$ for 5 days every 28 days) [5-17].

Prognosis and response rates with TMZ are known to be better in patients presenting with a methylated MGMT promoter gene (06-alkylguanine DNA alkyltransferase). Survival of patients with methylated MGMT is 21.7 months compared with 15.3 months for patients with a non-methylated gene. Recent clinical trials in elderly patients (more than 65 years of age) diagnosed with GB showed that TMZ is not inferior to radiotherapy. Patients with MGMT promoter methylation experienced the best results, facilitating decision-making in this fragile elderly population. There are no cures at present [18].

\section{On Glioblastoma Multiform}

\section{Morphology}

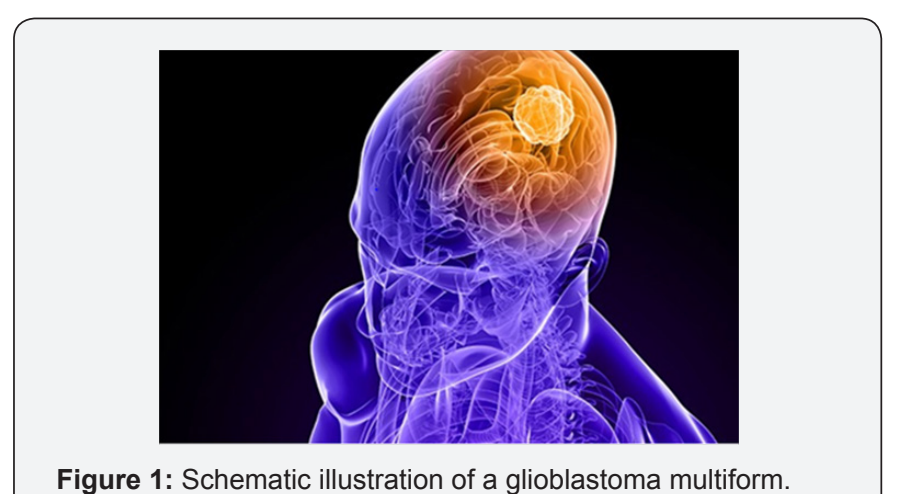

Figure 1: Schematic illustration of a glioblastoma multiform.

The tumor originates from primitive precursors of glial cells (glioblasts). Its highly variable appearance is due to the presence of necrosis, hemorrhage and cysts (multiform). Cancer cells with stem cell-like (SCLC) properties have been found in
GBs and this may be a cause of their resistance to conventional treatments as well as their high recurrence rate. These so-called GB stem-like cells (GBSCLC) reside in a niche around arterioles. The niche protects these cells against therapy by maintaining a relatively hypoxic environment. A biomarker for cells in GBs that exhibits cancer stem cell properties (the transcription factor HES3) has been shown to regulate their number when placed in culture [19-21]. Figures $1 \& 2$ provide a schematic illustration of Glioblastoma and its surgical resection.

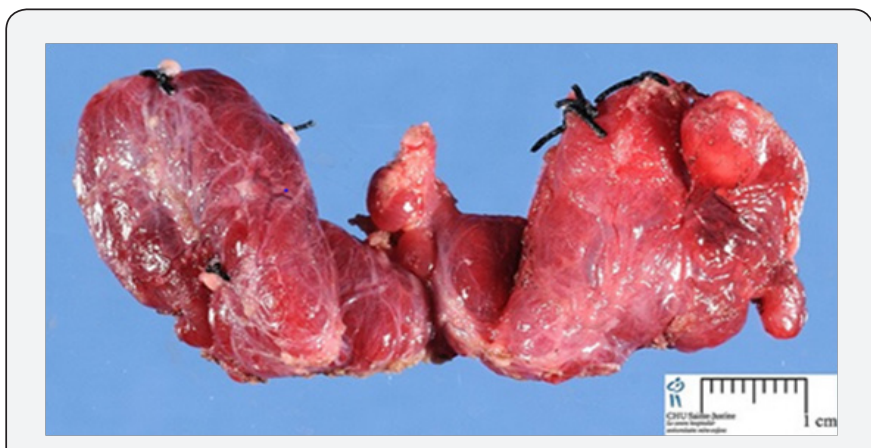

Figure 2: Surgical total resection of a Glioblastoma.

It is important to distinguish primary from secondary GBs, the former being the main subject of this article. Secondary (recurring) recurring tumors will be addressed in a companion article by this author. The primary tumors occur spontaneously or have progressed from a lower-grade glioma. Primary GBs have a worse prognosis, different tumor biology, and may have a different response to therapy, resulting in a critical evaluation to determine patient prognosis and therapy. Over $80 \%$ of secondary GBs carry a mutation in IDHI, whereas this mutation is rare in primary GBs (5-10\%). Thus, IDHI mutations are a useful tool to distinguish primary and secondary GBs since histopathologically they are very similar and the distinction without molecular biomarkers is unreliable.

Primary tumors: Of unclear cause, primary tumors can start from normal brain cells in the cerebral white matter then grow quickly, infiltrate small areas of the tumor, spread to other areas, and can become very large before producing symptoms (this is consistent with our current understanding of cancer as braided in our genome [15-21].

Secondary tumors: Less than $10 \%$ of GBs form slowly following degeneration of an existing low-grade astrocytoma (a brain tumor) or a plastic astrocytoma. These so-called secondary GBs are more common in younger patients (mean age 45 versus 64 years). The tumor may extend into the meninges or ventricular wall, leading to high protein content in the cerebrospinal fluid (CSF) ( $>100 \mathrm{mg} / \mathrm{dL}$ ), as well as an occasional pleocytosis of 10 to 100 cells (mostly lymphocytes). Malignant cells carried in the CSF may spread (rarely) to the spinal cord or cause meningeal gliomatosis. However, metastasis of GB beyond the CNS is extremely unusual. About $50 \%$ of GBs occupy more than one lobe of a hemisphere or are bilateral. Tumors of this 
type usually arise from the cerebrum and may exhibit the classic infiltration across the corpus callosum, producing a butterfly (bilateral) glioma. Most GB tumors appear to be sporadic, without any genetic predisposition.

\section{Biological Types}

\section{Molecular alterations}

Four subtypes of glioblastoma have been identified:

a) Classical: $90 \%$ of these tumors carry extra copies of the epidermal growth factor receptor (EGFR) gene, and most have higher than normal expression of EGFR. On the other hand, the gene TP53 (p53), which is often mutated in glioblastoma, is rarely mutated in this subtype.

b) Proneural: Often, this subtype has high rates of alterations of several genes: TP53 (p53), PDGFRA, GFR (encoding A-type platelet-derived GFR), and IDH1 (the gene encoding isocitrate dehydrogenase-1).

c) Mesenchymal: Characterized by high rates of mutations or other alterations in NF1 (the gene encoding Neurofibromin-1 and fewer alterations in the EGFR gene and less expression of EGFR than other types).

d) Neural: Was typified by the expression of neuron markers such as NEFL, GABRA-1, SYT-1 and SLC12A5.

\section{Genetic alterations}

Many other genetic alterations have been described in GBs. The majority of them are clustered in two pathways: RB and P13K/AKT with alterations in 68-78 \% and $88 \%$, respectively.

\section{Methylation of MGMT}

Another important alteration is methylation of MGMT (a "suicide" DNA repair enzyme), which impairs DNA transcription and, therefore, the expression of the MGMT enzyme. Since, due to its suicide repair mechanism, an MGMT enzyme can only repair one DNA alkylation, reverse capacity is low and methylation of the MGMT gene promoter greatly affects DNA-repair capacity. Indeed, MGMT methylation is associated with an improved response to treatment with DNA-damaging chemotherapeutics, such as TMZ.

\section{Risk Factors}

Several risk factors have been identified. These include:

1. Previous treatment with radiation therapy (a small link with ionizing radiation);

2. Lead exposure in the workplace;

3. Sex: male (for unknown reasons, GB is slightly more common in men than women);

4. Age: over 50 years old, most commonly around 64 years of age;
5. Ethnicity: Caucasians, Hispanics, and Asians;

6. Having a low-grade astrocytoma, which often given enough time develops into a higher-grade tumor; and

7. Genetic disorders such as neurofibromatosis (uncommon);

Certain genetic disorders associated with an increased incidence of gliomas:

a) Neurofibromatosis (types 1 and 2);

b) Tuberous sclerosis;

c) Von Hippel-Lindau syndrome;

d) Li Fraumeni syndrome; and

e) Turcot syndrome.

\section{Prognosis}

Criteria to assess progressions have been established by the Response Assessment in Neuro-Oncology (RANO) Working Group. Pseudo-progression is the result of radio necrosis, edema, and mass effect, simulating true progression. This is not uncommon in the first months after radiotherapy (incidence $\sim 20 \%-30 \%$ in patients who continue treatment with radio chemotherapy).

\section{Typical}

About 3 per 100,000 people develop the disease per year. Increasing age ( $>60$ years of age) carries a worse prognostic risk. Death is usually due to widespread tumor infiltration with cerebral edema and increased intracranial pressure. The median survival time from the time of diagnosis without any treatment is typically $\sim 3$ months, but with treatment survival of $\sim 1-2$ years is common. The most common length of survival following diagnosis is 12-15 months, with less than $3 \%$ to $5 \%$ of patients surviving longer than five years, although there are some long-term survivors [22].

\section{Longer survival}

Several indicative tests have been devised for predicting longer survival [23]:

a) Karnofsky Performance Score (KPS) and MGMT methylation: Both are associated with longer survival.

b) DNA: This test can be conducted on GBs to determine whether or not the promoter of the MGMT gene is methylated. Patients with a methylated MGMT promoter have longer survival than those with an unmethylated MGMT promoter, due in part to increased sensitivity to TMZ. This DNA characteristic is intrinsic to the patient and currently cannot be altered externally [22, 24-31].

c) IDHI gene mutation: This is a positive prognostic marker for GB patients. It can be tested by DNA-based 
methods or by immune histochemistry using an antibody against the most common mutation, namely IDH1-R132H.

d) Combination of IDHI mutational status and MGMT methylation status (a two-gene predictor): More prognostic power can be obtained by this combination. Patients with both IDH1 mutations and MGMT methylation have the longest survival, patients with an IDH1 mutation or MGMT methylation an intermediate survival, and patients without either genetic event have the shortest survival [29,31-33].

\section{Management and Treatment}

It is very difficult to treat GBs due to several complicating factors, including (a) the tumor cells are very resistant to conventional therapies, (b) the brain is susceptible to damage due to conventional therapy, (c) the brain has a very limited capacity to repair itself and (d) many drugs cannot cross the BBB to act on the tumor. Molecular tumor analysis (MTA) is used to prognosticate and group patients into more homogeneous groups to allow more rational choices of treatments and more rigorous clinical trials [40]. Treatment consists of both symptomatic and palliative therapies:

\section{Symptomatic Therapies}

Supportive treatment focuses on relieving symptoms and improving the patient's neurologic function. It employs:

i. Anticonvulsant corticosteroids: These are the primary supportive agents. Historically, $\sim 90 \%$ of patients with GB underwent anticonvulsant treatment, although only $40 \%$ of them required this treatment. It has been recommended that neurosurgeons not administer anticonvulsants prophylactically, and should wait until a seizure occurs before prescribing this medication.

ii. Phenytoin concurrent with radiation: Patients receiving this combination may have serious skin reactions such as erythema multiform and Stevens-Johnson syndrome.

iii. Corticosteroids: Usually Dexamethasone (dose regimen: 4-8 mg every 4-6 h) can reduce peritumoral edema through rearrangement of the $\mathrm{BBB}$, diminishing mass effect and lowering intracranial pressure with a decrease in headache or drowsiness.

\section{Palliative therapies}

Palliative treatment for GB consists of the standard triad (surgery followed by radiotherapy and chemotherapy, generally with TMZ). It is usually conducted to improve quality of life and achieve a longer survival time. (In a companion article, I will later address the case of recurrent GBs, an unfortunate occurrence in almost all cases). The UCLA Neuro-Oncology Department [33, 34] is the only U.S. institution that publishes real-time survival data on how its GB patients are performing. They also provide the list of chemotherapeutic agents they use to treat them. Nonetheless, despite a poor prognosis, a small number of survivors have been GB-free for more than 10-20 years. According to a 2003 study, GB prognosis can be divided into three subgroups dependent on KPS, patient age and treatment.

Table 1: Recursive Partitioning Analysis Versus Historical 5-year Survival (median, 1-, 3- and survival).

\begin{tabular}{|c|c|c|c|c|c|}
\hline $\begin{array}{c}\text { Recursive } \\
\text { partitioning } \\
\text { analysis (RPA) class }\end{array}$ & Definition & $\begin{array}{l}\text { Historical Median } \\
\text { Survival Time }\end{array}$ & $\begin{array}{l}\text { Historical 1-Year } \\
\text { Survival }\end{array}$ & $\begin{array}{c}\text { Historical 3-Year } \\
\text { Survival }\end{array}$ & $\begin{array}{c}\text { Historical 5-Year } \\
\text { Survival }\end{array}$ \\
\hline III & Age $<50$, KPS $\geq 90$ & 17.1 months & $70 \%$ & $20 \%$ & $14 \%$ \\
\hline \multirow[t]{2}{*}{ IV } & Age $<50$, KPS $<90$ & 11.2 months & $46 \%$ & $7 \%$ & $4 \%$ \\
\hline & $\begin{array}{c}\text { Age } \geq 50, \mathrm{KPS} \geq 70, \\
\text { surgical removal } \\
\text { with good neurologic } \\
\text { function }\end{array}$ & & & & \\
\hline \multirow{3}{*}{$V+V I$} & $\begin{array}{c}\text { Age } \geq 50, \mathrm{KPS} \geq 70 \\
\text { surgical removal } \\
\text { with poor neurologic } \\
\text { function }\end{array}$ & 7.5 months & $28 \%$ & $1 \%$ & $0 \%$ \\
\hline & $\begin{array}{c}\text { Age } \geq 50, \mathrm{KPS} \geq 70, \text { no } \\
\text { surgical removal }\end{array}$ & & & & \\
\hline & Age $\geq 50$, KPS $<70$ & & & & \\
\hline
\end{tabular}

Source: Stummer et al. [35].

Table 1 defines the recursive partitioning analysis (RPA) in terms of patient age and KPS, and whether surgery was either performed with good or poor neurologic function or not performed. It provides the RPA versus historical survival durations (median survival time, and percentages of 1,3 , and 5-year survivals). Two recent phase III clinical trials investigated the addition of Bevacizumab to standard treatment with TMZ.
They showed no increase in overall survival (OS) but diseasefree survival (DFS) increased. In addition, several clinical studies have looked at (a) the use of various drugs (e.g., integrin inhibitors such as Cilengitide and other antiangiogenics such as Cediranib) and (b) vaccines against EGFR, specifically EGFR variant 3 (EGFRV3), which is detected in $30 \%$ of patients. 


\section{Surgery}

\section{Basic approach}

Surgery is the first stage of GB treatment. After surgery, the average number of GB tumor cells is reduced by 98\%. Benefits of surgery include:

1. Resection for a pathological diagnosis;

2. Alleviation of symptoms related to mass effect; and potentially

3. Removing disease before secondary resistance to radiotherapy and chemotherapy occurs.

The greater the extent of tumor removal the better although it is not clear whether total resection is a requirement. Nonetheless, retrospective analyses indicate that removal of 98 $\%$ or more of the tumor has been associated with a significantly longer and healthier time, particularly if surgery is guided by a fluorescent dye. Because GB cells are widely infiltrative through the brain at diagnosis, and despite a "total resection" of all obvious tumor, recurrent GB tumors occur either near the original site or at more distant locations within the brain [35, 36](Figure 2).

\section{Improvements in surgical approaches}

Today, there have been substantial improvements in surgical approaches including:

1. New methods of detecting tumor anatomy with special illumination techniques [37];

2. A fluorescent dye (5-aminolevulinic acid) for identifying tumor cells in situ during surgery helps assure more complete excision of malignant tissue; and

3. New techniques in minimally invasive, image-guided surgery and intra-operative MRI [37], all of which are enabling surgeons to remove GBs with minimal morbidity (Figures 3 and 4).

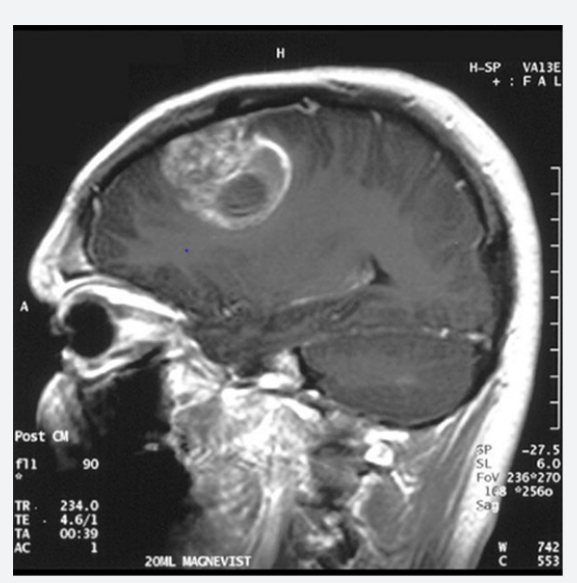

Figure 3: Computed tomography contrasted image of a Glioblastoma.

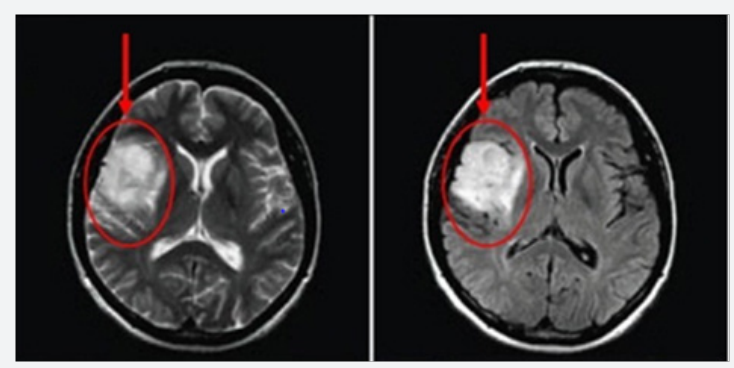

Figure 4: Sagittal computed tomography views of a Glioblastoma.

A maximally feasible resection with maximal tumor-free margins is usually performed along with external beam radiation and chemotherapy. Gross total resection of tumor is associated with a better prognosis although, as stated earlier, it is not clear whether removing the entire tumor or simply most of the cancer is preferable for, despite maximum treatment, the cancer usually recurs. High-dose steroids may be used to help reduce swelling and decrease symptoms.

\section{Complementary and Alternative Treatment Approaches}

Several other treatment approaches have been developed. These include:

\section{Conformal radiotherapy}

Subsequent to surgery, conformal (as opposed to whole brain) radiotherapy (CRT) combined with TMZ (standard schedule of $150-200 \mathrm{mg} / \mathrm{m} 2$ for 5 days every 4 weeks) is used to suppress and slow recurrent disease. Tumor margins can better be defined using magnetic resonance spectroscopy (MRS) for help in radiation treatment planning. On average, radiotherapy after complete resection surgery can reduce the tumor size by $\sim 98 \%$. A total radiation dose of 60-65 Gy has been found to be optimal for treatment. Nonetheless, GB tumors are well known to contain zones of tissue exhibiting hypoxia, which are highly resistant to radiotherapy.

\section{Boron neutron capture therapy}

Boron neutron capture therapy (BNCT) has been tested as an alternative treatment for GB but is not in common use [38].

\section{Intensity-modulated proton beam therapy}

Intensity-modulated proton beam therapy (IMPBT) has also been tested but likewise is not in common use.

\section{Chemotherapy}

Chemotherapy has historically provided little durable benefit as tumors recur within several months even in the case of the more accessible tumors located outside the brain. For brain tumors, access to the tumor is more challenging because of the presence of the brain protective barriers (BPBs), chiefly the blood brain barrier (BBB) $[6,7]$. 
Most studies show no benefit from chemotherapy alone. However, standard radiation plus TMZ chemotherapy allows a longer survival rate by $\sim 2.5$ months. This treatment regime is now standard for most cases of GB where the person is not enrolled in a clinical trial. TMZ seems to work by sensitizing the tumor cells to radiation. High doses of TMZ in high-grade gliomas yield low toxicity, but the results are comparable to the standard doses. Further, in an important development, TMZ is now a standard of care for all patients, including advanced age patients.

Various approaches to chemotherapy radio sensitizers such as the use of an oxygen diffusion-enhancing compound (ODEC), e.g., trans-sodium crocetinate (TSC), have been pursued with limited success.

\section{Antiangiogenic therapy}

GBs are highly vascular tumors and over-express endothelial vascular growth factor (EVGF, a pro-angiogenic cytokine). Premised on earlier studies that had shown promising radiographic response rates, delayed tumor progression, and a relatively safe profile for anti-EVGF agents, several randomized phase III trials were conducted. Unfortunately, to date, the trials have not shown a benefit in overall survival either in the case of antiangiogenic agents alone or in combination with chemo radiotherapy. In other words, antiangiogenic agents might not be beneficial in unselected populations of patients with GB [39].

Even for selected patients, biomarker identification has lagged behind the process of drug development, and no validated biomarker for patient stratification exists. Nonetheless, phase II trials have revealed an association between increased perfusion or oxygenation (these are consequences of vascular normalization) and survival. This suggests that early-imaging biomarkers could help to identify the subset of patients who will most likely benefit from anti-EVGF agents.

Recent experiments with mice have shown that cancer cells hijack and feed-off blood vessels. In the brain, these results in a weakened BBB and this may provide one reason for the rapid spread of GBs. If applicable to humans, this observation may lead to new ways to kill brain tumors with antiangiogenic therapy taking advantage of the BBB's weakness to get targeted drugs into the brain during the early stages of the cancer [40]. Antiangiogenic therapies with medications such as Bevacizumab control symptoms but do not affect overall survival [1, 3].

\section{Alternating electric field therapy}

Alternating intermediate frequency electric field therapy (AIFEFT) for several hours daily is a (U.S.) FDA-approved therapy for newly diagnosed and recurrent GBs. The device delivers electrical fields $(100-300 \mathrm{kHz})$ as an adjunct to standard treatment. Combined with TMZ in newly diagnosed GBs, this other treatment may improve by approximately three months the progression-free survival (PFS), and by five months the overall survival (OS) compared to TMZ therapy alone. The reported efficacy is similar to the modest results obtained with various schedules of second-line chemotherapy. Latest results show significant advantages in PFS and OS in first-line treatment. When used in combination with TMZ, PFS=7.1 months and OS=19.6 months as compared to 4 months and 16.6 months, respectively for TMZ alone. Despite these results, which could potentially change the first-line therapy standard, the efficacy of this approach remains controversial among medical experts and its second-line lacks a clear option $[4,41]$.

\section{The Optune tumor treating fields}

This is an electrical device that in one recent trial appeared to boost 5-year survival rate from $5 \%$ to $13 \%$.

\section{Immunotherapy}

The application of immunotherapy in the case of brain cancers has been discussed elsewhere [42-44].

\section{Vaccines}

A vaccine against cytomegalovirus has shown benefit for glioma patients in an early trial.

\section{Lifestyle changes}

Some researchers have advocated lifestyle changes as therapies that have no side effects and may be more efficient, including:

1. Vitamin D3 together with other antioxidants to reduce significantly and potentiate the effectiveness of chemotherapy, targeted therapy, and focused radiation, leading to fewer side effects; and

2. Ketosis diet to starve GBs and other tumors from needed glucose.

\section{Long Term Benefits}

Long-term benefits have been associated with those patients who receive surgery, radiotherapy, and TMZ chemotherapy. However, much remains unknown about why some patients survive longer than others with GB. Age under 50 is linked to longer survival; age under 40 is associated with a much better prognosis with a small fraction of patients achieving a population-based cure (PBC). The PBC is thought to occur when a population's risk of death (ROD) returns to that of the normal population and, in GB, this is thought to occur after 10 years. Likewise, $98 \%$ + resection and use of TMZ chemotherapy and better KPS are also linked to better GB survival.

\section{Summary and Conclusions}

Glioblastoma (or glioblastoma multiform) is the most common primary brain tumor in adults. It remains an unmet need in oncology. I have reviewed the difficulties encountered 
in devising treatment lines and the correspondingly associated risks. A distinction was made between a primary tumor and its metastases and the often recurring tumor, which I am deferring to a companion article. I have analyzed the various treatment options whether in isolation or in combination.

Chemotherapy (or treatment with cytotoxic drugs) has historically been not beneficial in general, and a fortiori not in the brain which is generally surrounded by protective barriers that present serious impediments to the delivery of therapeutic drugs. Over recent years, multiple drugs have been assessed both in monotherapy or combination therapy. First-line chemotherapeutic treatments employ the drug Temozolomide either alone or in combination with other drugs, nitrosoureas and alkalyting agents; antiangiogenic agents; epidermal growth factor receptor gene inhibitors; and several other agents.

In addition to chemotherapy, I have reviewed the other treatment strategies that are used either singly or in combination with chemotherapy, including: surgery, conformal radiotherapy, boron neutron therapy, intensity modulated proton beam therapy, antiangiogenic therapy, alternating electric fields, vaccines, palliative therapies and even lifestyle changes.

\section{References}

1. World Health Organization (2014) World Cancer Report 2014, Chapters 3.8 \& 5.16, Switzerland.

2. Dall'oglio S, D'Amico A, Pioli F, Gabbani M, Pasini F, et al. (2008) Doseintensity temozolomide after concurrent chemoradiotherapy in operated high-grade gliomas. J Neurooncol 90(3): 315-319.

3. Gallego $O$ (2015) Nonsurgical treatment of recurrent glioblastoma. Curr Oncol 22(4): e273-281.

4. Hart MG, Garside R, Rogers G, Stein K, Grant R (2013) Temozolomide for high grade glioma. Cochrane Database Syst Rev 4: CD007415.

5. Khosla D (2016) Concurrent therapy to enhance radiotherapeutic outcomes in glioblastoma. Ann Transl Med 4(3): 54.

6. Mason WP, Mirimanoff RO, Stupp R (2006) Radiotherapy with concurrent and adjuvant Temozolomide: A new standard of care for glioblastoma multiform. Progress in Neurotherapeutics and Neuropsychopharmacology 1(1): 37-52.

7. US National Cancer Institute (2007) Temozolomide plus Radiation helps brain cancer .

8. Niyazi M, Zehentmayr F, Niemöller OM, Eigenbrod S, Kretzschmar H, et al. (2011) MiRNA expression patterns predict survival in glioblastoma. Radiation Oncology 6(1): 153.

9. Stupp R, Mason WP, van den Bent MJ, Weller M, Fisher B, et al. (2005) Radiotherapy plus concomitant and adjuvant Temozolomide for glioblastoma. N Engl J Med 352(10): 987-996.

10. Stupp R, Taillibert S, Kanner AA, Kesari S, Steinberg DM, et al. (2015) Maintenance therapy with tumor-treating fields plus Temozolomide vs Temozolomide alone for Glioblastoma. JAMA 314(23): 2535-2543.

11. Smoll NR, Schaller K, Gautschi OP (2012) The cure fraction of Glioblastoma multiform. Neuroepidemiology 39(1): 63-69.

12. Ghebeh H, Bakr MM, Dermime S (2008) Cancer stem cell immunotherapy: The right bullet for the right target. Hematol Onco
Stem Cell Ther 1(1): 1-2.

13. Moviglia GA, Carrizo AG, Varela G, Gaeta CA, Paes de Lima A, et al (2008) Preliminary report on tumor stem cell/B cell hybridoma vaccine for recurrent Glioblastoma multiform. Hematol Oncol Stem Cell Ther 1 (1): 3-13.

14. Stuckey D, Hingtgen S, Karaka N, Rich B, Shah K (2014) Engineering toxin-resistant therapeutic stem cells to treat brain tumors. Stem Cells 33: 589-600.

15. Fymat AL (2016) Recent research developments in anti-cancer therapy. J Cancer Prev Curr Res 5(2): 1-2.

16. Fymat AL (2016) Nanotechnology and cancer. J Cancer Prev Curr Res $5(6): 1-7$.

17. Fymat AL (2016) The long quest for cancer cures. J Cancer Prev Curr Res 6(2): 1-3.

18. Fymat AL (2017) On cancer electro- and nano-chemotherapy. J Cancer Prev Curr Res 7(2): 1-4.

19. Fymat AL (2017) Anti-Tumor Therapies-Cases of Breast and Prostate Cancers. J Tumor Medicine \& Prevention 1(2)1-12.

20. Fymat AL (2017) Nanoneurology-Drug delivery across the brain protective barriers. J Nanomed Res 5(1): 1-4.

21. Fymat AL (2017) Therapeutics delivery behind, through and beyond the blood brain barrier. Open Access Journal of Surgery 5 (1): 1-9.

22. Showalter TN, Andrel J, Andrews DW, Curran WJ, Daskalakis C, et al. (2007) Multifocal Glioblastoma Multiform: Prognostic Factors and Patterns of Progression. Int J Radiat Oncol Biol Phys 69(3): 820-824.

23. Showalter TN, Andrel J, Andrews DW, Curran WJ, Daskalakis C (2007) Multifocal Glioblastoma Multiform: Prognostic Factors and Patterns of Progression. Int J Radiat Oncol Biol Phys 69(3): 820-824.

24. Fymat AL (2017). Genetics, epigenetics and cancer. Cancer Therapy \& Oncology Int J 4(2): 1-11.

25. Hayden EC (2010) Genomics boosts brain-cancer work. Nature 463(7279): 278.

26. Kuehn BM (2010) Genomics Illuminates a Deadly Brain Cancer. JAMA 303 (10): 925-927.

27. Ohgaki $H$, Kleihues $P$ (2009) Genetic alterations and signaling pathways in the evolution of gliomas. Cancer Sci 100(12): 2235-2241.

28. Verhaak RG, Hoadley KA, Purdom E, Wang V, Qi Y, et al. (2010) Integrated genomic analysis identifies clinically relevant subtypes of glioblastoma characterized by abnormaiities in PDGFRA, IDH1, and NF1. Cancer Cell 17(1): 98-110.

29. Hegi ME, Diserens AC, Gorlia T, Hamou MF, de Tribolet N, et al. (2005) MGMT Gene silencing and benefit from Temozolomide in Glioblastoma. N Engl J Med 352(10): 997-1003.

30. Martinez R, Schackert G, Yaya-Tur R, Rojas-Marcos I, Herman J, et al. (2006) Frequent hypermethylation of the DNA repair gene MGMT in long-term survivors of glioblastoma multiform. Journal of NeuroOncology 83 (1): 91-93.

31. Molenaar RJ, Verbaan D, Lamba S, Zanon C, Jeuken JW, et al. (2014) The combination of IDH1 mutations and MGMT methylation status predicts survival in glioblastoma better than either IDH1 or MGMT alone. Neurooncology 16(9): 1263-1273.

32. Molenaar RJ, Verbaan D, Lamba S, Zanon C, Jeuken JW, et al. (2014) The combination of IDH1 mutations and MGMT methylation status predicts survival in glioblastoma better than either IDH1 or MGM alone. Neuro-Oncology. 16(9): 1263-1273. 
33. Krex D, Klink B, Hartmann C, von Deimling A, Pietsch T, et al. (2007) Long-term survival with glioblastoma multiform. Brain 130(10): 2596-2606

34. Shaw EG, Seiferheld W, Scott C, Coughlin C, Leibel S, et al. (2003) Reexamining the radiation therapy oncology group (RTOG) recursive partitioning analysis (RPA) for glioblastoma multiform (GBM) patients. International Journal of Radiation Oncology Biology Physics 57(2): S135-136.

35. Stummer W, Pichlmeier U, Meinel T, Wiestler OD, Zanella F, et al (2006) Fluorescence-guided surgery with 5-aminolevulinic acid for resection of malignant glioma: A randomized controlled multicenter phase III trial. Lancet Oncol 7 (5): 392-401.

36. Young RM, Jamshidi A, Davis G, Sherman JH (2015) Current trends in the surgical management and treatment of adult glioblastoma. Ann Transl Med 3(9): 121.

37. Fymat AL (2017) Magnetic resonance imaging modalities with contrast enhancing materials. Current Trends in Clinical \& Medical Imaging 1(1): 1-4.

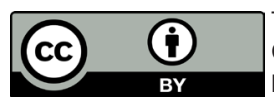

This work is licensed under Creative Commons Attribution 4.0 Licens

DOI: 10.19080/OAJS.2017.07.555706
38. Yang, Guo G, Niu XY, Liu J (2015) Dendritic cell-based immunotherapy treatment for Glioblastoma multiform. Biomed Res Int 2015: 717530.

39. Fymat AL (2017) Antiangiogenic targeting of early developing glioblastomas behind a weakened blood brain barrier (preprint) 2(3): 1-6.

40.Sampson JH (2015) Alternating electric fields for the treatment of glioblastoma. JAMA 314(23): 2511.

41. Fymat AL (2017) Immunotherapy: An emergent anti-cancer strategy. Journal of Cancer Prevention \& Current Research 7(3): 1-4.

42. Fymat AL (2017) Synthetic immunotherapy with chimeric antigen receptors. Journal of Cancer Prevention \& Current Research 7(5): 1-3.

43. Fymat AL (2017) Immunotherapy of brain cancers and neurological disorders. J Cancer Prev Curr Res 2017 (preprint) 8(6): 1-7.

\section{Your next submission with Juniper Publishers will reach you the below assets}

- Quality Editorial service

- Swift Peer Review

- Reprints availability

- E-prints Service

- Manuscript Podcast for convenient understanding

- Global attainment for your research

- Manuscript accessibility in different formats

( Pdf, E-pub, Full Text, Audio)

- Unceasing customer service

Track the below URL for one-step submission https://juniperpublishers.com/online-submission.php 